\title{
Rituximab Induced Interstitial Lung Disease Diagnosis, Treatment Outcome, and Risk's Factor, a Place for Transbronchial Pulmonary Cryobiopsy
}

\section{Douze Makenzi' ${ }^{1}$ Vintila Sabina ${ }^{2}$, Jossart Adrien³, Praile Martin'3, Klipper Dit Kurtz Noemie ${ }^{3}$, Makhoul Martin ${ }^{3}$, Mond Victoria ${ }^{3}$, Maeck Patricia ${ }^{4}$}

${ }^{1}$ Department of Nephrology, Hospital Center of Mouscron, Mouscron, Belgium

${ }^{2}$ Department of Pneumology, Hospital Center of Mouscron, Mouscron, Belgium

${ }^{3}$ Department of Interne Medicine, Hospital Center of Mouscron, Mouscron, Belgium

${ }^{4}$ Department of Onco-Hematology, Hospital Center of Mouscron, Mouscron, Belgium

Email: douzemakensie@yahoo.fr

How to cite this paper: Makenzi, D., Sabina, V., Adrien, J., Martin, P., Noemie, K.D.K., Martin, M., Victoria, M. and Patricia, M. (2021) Rituximab Induced Interstitial Lung Disease Diagnosis, Treatment Outcome, and Risk's Factor, a Place for Transbronchial Pulmonary Cryobiopsy. Case Reports in Clinical Medicine, 10, 284-294.

https://doi.org/10.4236/crcm.2021.1010036

Received: August 19, 2021

Accepted: October 17, 2021

Published: October 20, 2021

Copyright $\odot 2021$ by author(s) and Scientific Research Publishing Inc. This work is licensed under the Creative Commons Attribution International License (CC BY 4.0).

http://creativecommons.org/licenses/by/4.0/

\begin{abstract}
Rituximab (RTX) is a mouse/human chimeric anti-CD20 IgG1 monoclonal antibody, approved in late 1998 by the FDA. Effectively used as a single agent or combined with a chemotherapy regimen to treat lymphoma, RTX is a significant step forward in the arsenal treatment of idiopathic thrombocytopenic purpura, systemic lupus erythematous, rheumatoid arthritis, and autoimmune hemolytic anemia. Side effects of RTX are commonly seen during the first infusion in up to $50 \%$ of patients and include fever, chills, and rigors. These side effects are generally transient and related to the tumor burden, probably due to a greater degree of complement activation and proinflammatory cytokine release. Severe lung toxicity like cryptogenic organizing pneumonia, pneumonitis, and interstitial lung diseases are infrequent, with most of the knowledge coming from case reports.
\end{abstract}

\section{Keywords}

Drug Induced Lung Disease, Histology/Cytology, ANCA Related Vasculitis, Drug Reactions, Rituximab

\section{Material and Methods}

The objective of this article is to peculiarize the syndrome of rituximab-induced interstitial lung disease (R-ILD). We proposed a thorough description of R-ILD, 
based on information gathered from reported cases and the medical literature reviews. Our emphasis will be on patients' characteristics, treatment options, clinical presentation, risks factors, imaging studies, and pulmonary function tests (PFTs); standard and new diagnostic procedure such Transbronchial Pulmonary cryobiopsy (TPC) will also be discussed. In a practical manner, we propose to do so by presenting two such cases.

\section{Introduction}

\subsection{Case One}

A 67-year-old male Caucasian patient presented at the emergency department with a history of progressive dyspnea, fever, and dry cough. Two weeks before the onset of his symptoms, he received his second dose of rituximab (RTX-based regimen of $1 \mathrm{~g}$ followed 14 days later of another $1 \mathrm{~g}$ dose) in the setting of a P-ANCA granulomatosis with polyangiitis diagnoses by renal biopsy. At the beginning of his condition, the patient presented with isolated renal involvement such as acute kidney injury, strong sediment hematuria, and nephrotic syndrome, $3.5 \mathrm{~g} / 24 \mathrm{hr}$. No other systemic presentations nor functional and radiological respiratory impairment were highlighted.

At the emergency department, his initial vital signs were as follows: temperature $36.9^{\circ} \mathrm{C}$, with a heart rate of 90 beats $/ \mathrm{min}$, blood pressure $140 / 80 \mathrm{mmHg}$, tachypnea with a respiratory rate of $20 / \mathrm{min}$ and oxygen saturation $86 \%$ on room air, $91 \%$ with $3 \mathrm{~L}$ of $\mathrm{O}_{2}$ within a nasal canula. Physical examination was unremarkable except for bilateral scattered inspiratory crackles.

Laboratory examination revealed normocytic anemia, a mild inflammatory syndrome, stable CKD (chronic kidney disease) function. A whole review is then launched; because of the current context, during a viral pandemic at SARS CoV2, a nasopharyngeal smear is performed to search for SARS CoV2 by polymerase chain reaction (PCR) method, which returns negative twice. The extend of other laboratory tests searching for an infectious etiology was negative (Table 1). Computer tomography (CT-Scan) of the chest revealed diffuse bilateral ground-glass opacities, poorly defined centrilobular nodules, and mosaic attenuation (Figure 1). Cardiac origin was excluded by a $2 \mathrm{D}$ echocardiogram that demonstrated an ejection fraction of $55 \%$ with no valvular abnormalities.

\section{Differential Diagnosis}

CT scan images finding, with a diffuse interstitial pathology aspect can be found on Nonspecific Interstitial Pneumonia (NSIP) and can either reflect extrinsic allergic alveolitis, interstitial hypersensitivity pneumonitis, toxic pneumonia, or diffuse infectious lung disease. Compared to the same review conducted for the GNSA's assessment, these lesions are new. Bronchoscopy with bronchoalveolar lavage (BBL) with transbronchial biopsy is mandatory to rule out an infection etiology, autoimmune features, or presences of malignancy cells. BBL was performed, with a negative result for the presence of SARS CoV2 or other infectious (Table 2), as such of atypic cells. A thorough review of the patient's 
Table 1. Laboratory results.

\begin{tabular}{ccc}
\hline Test & Patient value & Normal range \\
\hline CPR & $16.8 \mathrm{mg} / \mathrm{dL}$ & $<5 \mathrm{mg} / \mathrm{dl}$ \\
BUN & $186 \mathrm{mg} / \mathrm{dL}$ & $17-43 \mathrm{mg} / \mathrm{dL}$ \\
Créatinin & $1.97 \mathrm{mg} / \mathrm{dL}$ & $0.6-1.3 \mathrm{mg} / \mathrm{dL}$ \\
$\mathrm{Na}$ & $137 \mathrm{mmol} / \mathrm{L}$ & $136-145 \mathrm{mmol} / \mathrm{L}$ \\
$\mathrm{K}$ & $4.8 \mathrm{mmol} / \mathrm{L}$ & $3.5-5.1 \mathrm{mmol} / \mathrm{L}$ \\
$\mathrm{Cl}^{-}$ & $99 \mathrm{mmol} / \mathrm{L}$ & $98-107 \mathrm{mmol} / \mathrm{L}$ \\
Ca & $2.3 \mathrm{mmol} / \mathrm{L}$ & $2.2-2.6 \mathrm{mmol} / \mathrm{L}$ \\
GOT-ASAR & $46 \mathrm{U} / \mathrm{L}$ & $<37.0 \mathrm{U} / \mathrm{L}$ \\
GPT-ALT & $48 \mathrm{U} / \mathrm{L}$ & $<45.0 \mathrm{U} / \mathrm{L}$ \\
Gamma-GT & $43 \mathrm{U} / \mathrm{L}$ & $<60.0 \mathrm{U} / \mathrm{L}$ \\
Hb & $11.7 \mathrm{~g} / \mathrm{dL}$ & $14.0-18.00 \mathrm{~g} / \mathrm{dL}$ \\
WBCs & $8.02 \times 1000 / \mathrm{mm}{ }^{3}$ & $4.0-10.0 \times 1000 / \mathrm{mm}{ }^{3}$ \\
Total Proteins & $60 \mathrm{~g} / \mathrm{L}$ & $60-80 \mathrm{~g} / \mathrm{L}$ \\
Albumine & $33.0 \mathrm{~g} / \mathrm{L}$ & $32-46 \mathrm{~g} / \mathrm{L}$ \\
Protein electrophoresis & Normale & Normale \\
ANCA & Positif & négative \\
Anti-MPO & $0.3 \mathrm{UI} / \mathrm{mL}$ & $<3.5 \mathrm{UI} / \mathrm{mL}$ \\
Anti-PR3 & $0.3 \mathrm{UI} / \mathrm{mL}$ & $\mathrm{UI} / \mathrm{mL}$ \\
\hline
\end{tabular}

Table 2. Bronchoalveolar lavage, cellular components.

\begin{tabular}{ccc}
\hline Cells types & Valeur du patient & Norme \\
\hline Macrophages & $17 \%$ & $85 \%$ \\
Lymphocytes & $66 \%$ & $10 \%-15 \%$ \\
Neutrophils & $17 \%$ & $<3 \%$ \\
Eosinophils & $0 \%$ & $<1 \%$ \\
Atypical cells & 0 & 0 \\
Culture & Negative & \\
PCR-SARS CoV2 Méthode CMIA-Abbott & negative & \\
\hline
\end{tabular}

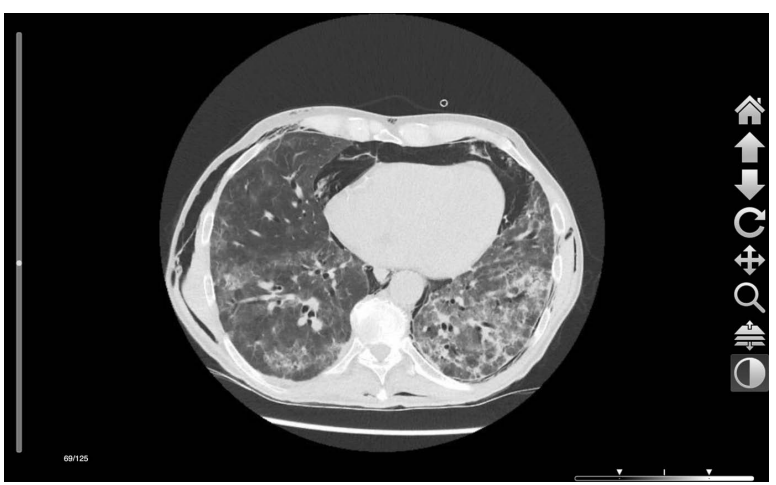

Figure 1. Computer tomography (CT-Scan) of the chest revealed diffuse bilateral ground-glass opacities, poorly defined centrilobular nodules, and mosaic attenuation. 
environmental and occupational exposure showed no apparent external cause for hypersensitivity pneumonia.

The favorable evolution of the GPA with the disappearance of anti-MPO antibodies, besides the absence of alveolar hemorrhage, does not sustain a vasculitis involving lung damage. Spirometry objectified a restrictive syndrome with a decrease of the FVC, TLC, and DLCO. Following this broad assessment, the diagnosis of interstitial lung disease remains the clearest etiology. Nevertheless, the exclusion of pulmonary involvement in the setting of GNSA is challenging in this context. Therefore, in the absence of any counterindications, a TPC is performed. The histologic report revealed strongly suggestive images of nonspecific interstitial pneumonia with enlarging septa, discrete fibrosis, and inflammatory infiltrates (Figure 2). All these findings support the diagnosis of interstitial lung disease (ILD).

Initially, pulse therapy, $250 \mathrm{mg}$ /day of methylprednisolone for three to five days. The patient presented immediate signs of clinical improvements, followed by oral prednisone at a dose of $1 \mathrm{mg} / \mathrm{kg}$ per day (using ideal body weight). Subsequent tapering down over six months was conducted with an excellent clinical

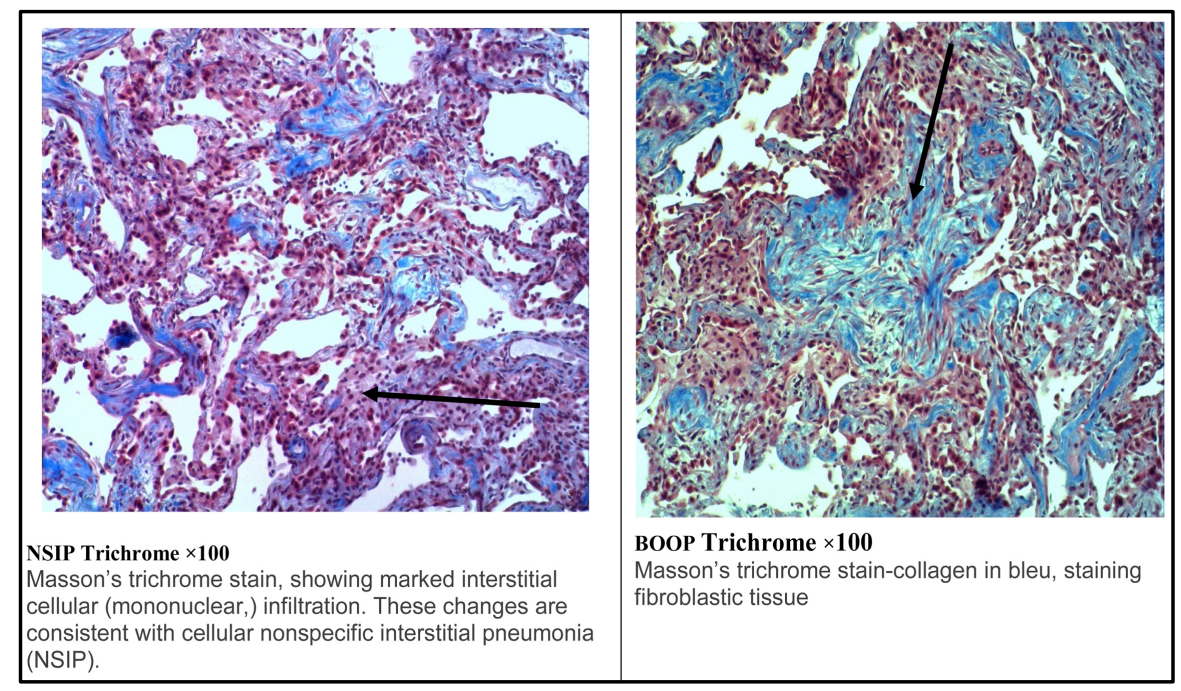

Figure 2. Histologic result. NSIP: Nonspecific interstitial pneumonia. BOOP: an Organizing pneumonia (previously called bronchiolitis obliterans organizing pneumonia. Histologic Result: moderate to severely increased pulmonary parenchyma stiffness, varying mononuclear cell inflammation degrees within the interalveolar septum. Presence of local patchy pattern accumulations of fibroblasts in some alveoli, also involving alveolar ducts. No evidence of dense fibrosis neither lymphoid follicles. No vasculitis images, no malignancy aspect identified. Immunostaining: TTF1 et KERAE3 confirmed enlargement of the septum and the interalveolar spaces, alveoli hyperplasia. No vasculitis images, no malignancy aspect identified. Trichrome stain confirmed accumulations of collagen-embedding fibroblasts foci in some of the interalveolar septum. Conclusion: histologic images suggest the presence of a Nonspecific interstitial pneumonia (NSIP) by the presence of an enlargement of the septum and the interalveolar spaces, alveoli hyperplasia, mononuclear cell inflammation. Some features also suggest the presence of an organizing pneumonia, OP (previously called bronchiolitis obliterans organizing pneumonia or BOOP). BOOP can be seen in combination with the interstitial cellular changes of NSIP in cases of drug-toxicity. 
and functional response.

\subsection{Case Two}

A 65-year-old man diagnosed with stage IV follicular lymphoma received two cycles of RTX and bendamustin with an interval of 2-week. Prior to initiation therapy lung examination was normal and a CT of the chest showed no lung abnormalities, (Case two CT scan's evolution Figure 3). He attended to our emergency department, with dry cough, fever, dyspnea, within 4 weeks after the completion of the second treatment cycle. Lung examination revealed diffuse bilateral scattered inspiratory crackles, tachycardia, tachypnea, and hypoxemia requiring $3 \mathrm{~L}$ of oxygen. Laboratory test showed a white blood cell count of $5900 / \mathrm{mm}^{3}$ (65.9\% neutrophils, $24.3 \%$ lymphocytes and $1.4 \%$ eosinophils), hemoglobin $11.2 \mathrm{~g} / \mathrm{dl}$, platelet count $243,000 / \mathrm{mm}^{3}$, blood urea nitrogen $40 \mathrm{mg} / \mathrm{dl}$, creatinine $1.2 \mathrm{mg} / \mathrm{dl}$, calcium $10.4 \mathrm{mg} / \mathrm{dl}$ and glucose $92 \mathrm{mg} / \mathrm{dl}$. Chest radiography showed diffuse bilateral lung infiltrates a computed tomography of the chest revealed diffuse bilateral ground-glass opacities, poorly defined centrilobular nodules and mosaic attenuation. Spirometry demonstrated a restrictive pattern with very low diffusion capacity (DLco 26\%).

A positron emission tomography (PET-SCAN) is requested as part of unexplained hypercalcemia to exclude a malignant tumor and bone metastases and sarcoidosis but did not reveal any malign lesion and ruled out any extra-pulmonary pathological suspicious abnormalities (Figure 4).

The patient underwent bronchoscopy with bronchoalveolar lavage and transbronchial biopsies bronchoscopy. Bronchoalveolar lavage fluid resumed lymphocytic alveolitis. All bacterial, viral, viral (including SARS-COV2) carinii pneumocystis, mycobacteria's and fungous were negative (Table 3). But no sufficient material was obtained from the transbronchial biopsies to identify a histologic entity.

A multidisciplinary consensus agrees to perform a (TLC) which showed desquamation of alveolar pneumocytes eosinophilic inflammation and macrophages with reactive changes consistent with alveolar damage interstitial fibrosis. Drug induced pneumonitis was the most likely possible underlying etiology. The diagnosis of RTX-ILD was made on clinical, radiological, and histological bases.

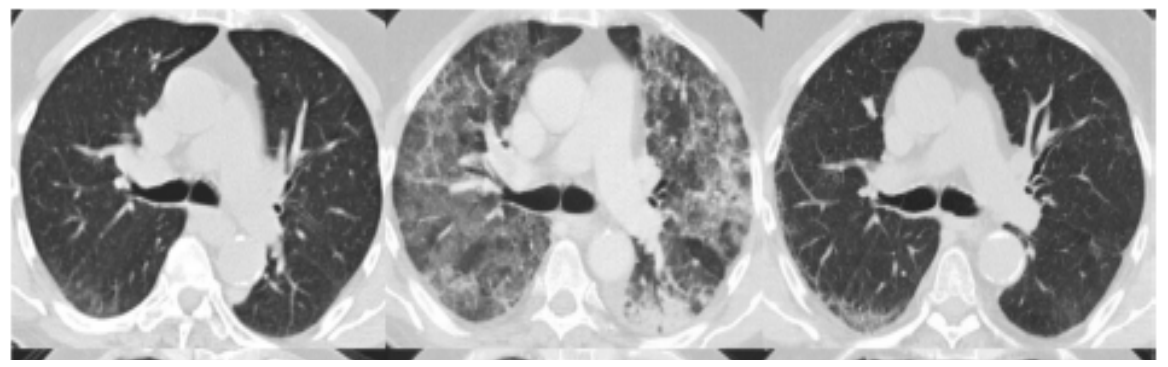

Figure 3. Case two CT scan's evolution. CT of the lungs prior to treatment with rituximab (left panel), during treatment with rituximab (middle panel), and after interruption of rituximab and initiation of corticoids treatment. 


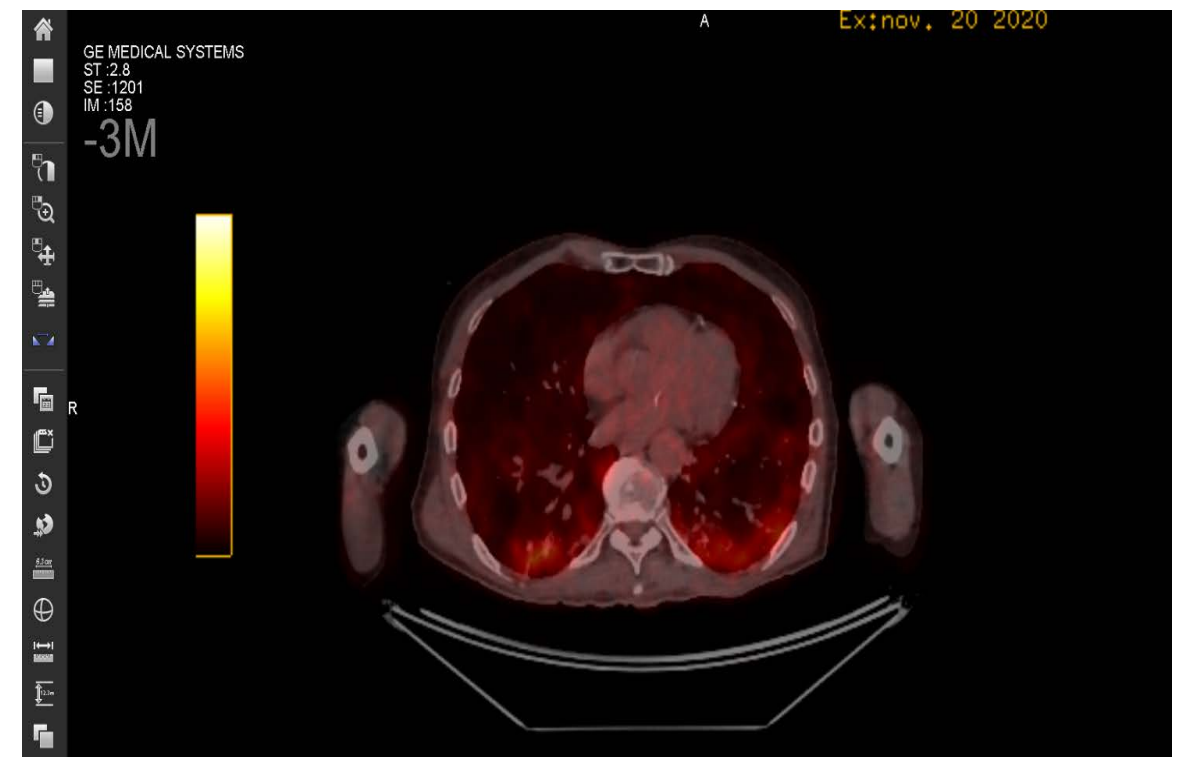

Figure 4. Positron emission tomography (PET-SCAN): highlighted a consistent fixation of the FDG, at the level of the lower lobes, and the presence of metabolically active pulmonary infiltrates (FDG SUV max: $6.7 \mathrm{~g} / \mathrm{ml}$ ) mainly in the posterior but also at the tops next to lesions in a ground glass as well as of the pleura. No malign lesions identified.

Table 3. Infectious panel.

\begin{tabular}{cl}
\hline Test & Patient results \\
\hline PCR and Sérologie SARS-CoV-2: SARS-CoV-2 IgG & Négative \\
HSV 1 \& 2 IgM (CMIA) & Négative \\
HZV IgM (CMIA) & Négative \\
Parvovirus B19: Parvovirus IgM & Négative \\
Parvovirus B19: Parvovirus IgG & Positive \\
Mycoplasma sp: Mycoplasma pneumonia-IgM & Negative \\
Mycoplasma pneumonia-IgG: $<0.100 \mathrm{UA} / \mathrm{mL}$ & Negative \\
Ag Aspergillus & Negative \\
Cytomégalovirus: C.M.V. IgM & Négative \\
\hline
\end{tabular}

We started Bolus of $250 \mathrm{mg} /$ day of methylprednisolone for three to five days. Within $24 \mathrm{hr}$ his condition dramatically improved. He was switched to oral prednisone at a dose of $60 \mathrm{mg}$ per day. The patient was discharged home with supplemental oxygen and tapering doses of steroids. Two months after hospital admission, patient follow up showed consistent improvement, oxygen supplement was gradually diminished, and withdrawn. A repeat CT scan of the chest showed almost complete resolution of his interstitial disease. Pulmonary function tests revealed considerable improvement in DLco to $48 \%$, which was the same level prior to initiation of RTX therapy a mild restrictive pattern with a mild diffusion defect. 


\section{Risk Factors and Patients' Characteristics for Rituximab-Induced Interstitial Lung Diseases}

\subsection{Epidemiology}

The incidence rate of RTX-ILD is unknown but rare; early reports indicated meager incidence rates at $0.010 .03 \%$ [1]. Nevertheless, much higher incidence rates were reported in post-marketing case series ranging from $3.7 \%$ to $10 \%$ [2]. Those discrepancies might be attributed to the difference in the target population between clinical trials and daily clinical practice. Besides, some cases of RTX-ILD might be regarded as lower respiratory tract infections because of the overlap between the signs and symptoms of these complications.

In a retrospective study of 264 patients with non-Hodgkin's lymphoma treated with rituximab-containing chemotherapy regimens, 9 (3.5\%) were diagnosed with rituximab-induced ILD, including clinically suspected cases $(n=5)$.

\subsection{Clinical Settings, Timing and Images Characteristics}

Details of RTX-ILD have been described in a systematic review [3], and the most common symptoms are dry cough, exertional dyspnea, and fever. Nonspecific symptoms are less common and included fatigue, rigors, wheeze, hemoptysis, skin rash, and pleuritic chest pain. Hadjinicolaou AV and al; in a case series, found that around $20 \%$ of patients were asymptomatic at the time of diagnosis, with the disease being detected either by CT or LFT. Three clinical presentations were identified based on symptoms onset: Early onset hyperacute forms (less than 7 days) after infusion. Acute/Subacute (7 to 21 days), Chronic (over 30 days) [4].

In a multivariate analysis, poor Eastern Cooperative Oncology Group (ECOG) performance status (odds ratio $10.8 \%$ and $95 \%$ confidence interval $1.6-74.8, \mathrm{p}=$ 0.016 ) and age (odds ratio $1.1 \%$ and $95 \%$ confidence interval $0.0-1.2, p=0.048$ ) were significant risk factors for rituximab-induced ILD. The most common form on CT scan is an organizing pneumonia pattern or/and diffuse interstitial pattern (ground-glass opacities, alveolitis, and diffuse infiltrate); diffuse or patchy bilateral consolidation in some cases had been described, with the addition of centrilobular nodules which can indicate the presence of alveolitis. Pleural effusion is uncommon; its presence a co-existing infection should be excluded. Hypersensitivity pneumonitis, ARDS, interstitial pneumonitis, organizing pneumonia, also had been reported [5]. Our findings are consistent with the reported cases in the literature in which alveolitis, fibrosis [6].

\subsection{Risks Factors}

The majority of patients had a diagnosis of NHL (75\%), and Hadjinicolaou et al. [1] suggested that the elderly is at the most significant risk of rituximab-associated IL D were elderly (average age 65). In the same multivariate analysis, poor Eastern Cooperative Oncology Group indicates that poor ECOG performance status and age are significant predictors of RX-ILD. The average number of cycles of 
rituximab before the presentation was four. Low serum albumin level was reported as a risk factor for adverse pulmonary reactions associated with the use of monoclonal antibodies in cancer patients [1]. A prospective longitudinal study conducted did not find any statistically significant rlation in smokers, nether underlying chronic lung disease, or exposure to occupational hazards. Pulmonary function tests have usually shown a reduction in the diffusion capacity (DLCO) in the range of DLCO values $13 \%$ - 33\% of predicted and restrictive patterns. Lung biopsy is not usually performed in RTX-ILD, but pulmonary inflammation is a standard feature [4]. The most consistent Lung biopsy in systematic review studies showed histological patterns of organizing pneumonia, interstitial pneumonitis, desquamative interstitial pneumonia, diffuse alveolar damage [6].

\subsection{Treatment Strategy}

Conventionally the treatment of RTX-ILD follows the general principles of drug-induced pulmonary toxicity: 1) discontinuation of the offensive agent, 2) supportive treatment, and 3) corticosteroid treatment. Steroids can be considered in patients with moderate-to-severe dyspnea, respiratory failure, and severe decline in lung function, particularly the diffusion capacity. No recommendations can be made about the dose, route of administration, or duration of steroid therapy.

\section{Discussion}

Rituximab-induced interstitial lung disease is a rare but known complication. Its low incidence may be attributed to a failure to recognize the complication or due to it spontaneously resolution after discontinuing the medication or after a steroid course [7].

The definite causal relationship is difficult to prove, but chronological association together with the described clinical and radiological features make a probable diagnosis of RTX-ILD. Another challenge in the diagnosis is the use of concomitant drugs or chemotherapeutic agents particularly in the treatment of lymphoma. For this dilemma some authors used Drug lymphocyte stimulation test, a result strongly positive for rituximab with elevated Levels of TNF-a, interferon $\mathrm{g}$, and interleukin 4 but negative for others immunosuppressive or chemotherapeutic agents (such as cyclophosphamide and vincristine) can be a useful tool to discriminate [8].

Bronchoalveolar lavage findings may support the likelihood of certain lung diseases; however, the information is not specific, and to obtain a definitive diagnosis, further investigations are required such as Transbronchial biopsy. Other alternative to enhance the diagnostic sensitivity in case of transbronchial biopsy failure could be the realization of a Transbronchial Pulmonary cryobiopsy. This relatively new technic has proved to be reasonably safe and useful as an evaluable tool in the pathological assessment of ILDs [9] [10]. 


\section{Conclusions}

Rituximab-induced interstitial lung disease is a rare pathology and often misdiagnosed. Clinical, laboratory and radiological findings can be inconstant, which can make its diagnosis quite challenging. Strong suspicions, clinic presentation, and well-trained clinicians can help an early diagnostic establish a corticotherapy regimen to prevent life-threatening complications.

TPC realization can be considered, this procedure has been proved to be a safe alternative to surgical lung biopsy in ILD diagnosis. Although this technique may not be applied to all ILD cases, it needs to be included in the armamentarium of techniques available for sampling lung tissue when deemed indicated.

\section{Statements}

This work was not supported by any grant or funding. We did not receive any contributions fees. There are no competing interests for any other participants (such as board membership, consultancy, employment, expert testimony grant, contract research, lectures/other education events). We do not require and exclusive license for this article publication. This article does not involve human participants neither any drugs trial.

\section{Conflicts of Interest}

The authors declare no conflicts of interest regarding the publication of this paper.

\section{References}

[1] Hadjinicolaou, A.V., Nisar, M.K., Parfrey, H., Chilvers, E.R. and Ostor, A.J. (2012) Non-Infectious Pulmonary Toxicity of Rituximab: A Systematic Review. Rheumatology, 51, 653-662. https://doi.org/10.1093/rheumatology/ker290

[2] Liu, X., Hong, X.N., Gu, Y.J., Wang, B.Y., Luo, Z.G. and Cao, J. (2008) Interstitial Pneumonitis during Rituximab-Containing Chemotherapy for Non-Hodgkin Lymphoma. Leukemia \& Lymphoma, 49, 1778-1783. https://doi.org/10.1080/10428190802270886

[3] Krishnaswamy, U.M. and Maka, V.V. (2014) Rituximab Induced Interstitial Lung Disease in Patients with Non-Hodgkin's Lymphoma: A Clinical Study of Six Cases and Review of the Literature. Clinical Study, 2014, Article ID: 160421. https://doi.org/10.1155/2014/160421

[4] Lioté, H., Lioté, F., Séroussi, B., Mayaud, C. and Cadranel J. (2010) Rituximab-Induced Lung Disease: A Systematic Literature Review. European Respiratory Journal, 35, 681-687. https://doi.org/10.1183/09031936.00080209

[5] Biehn, S.E., Kirk, D., Rivera, M.P., Martinez, A.E., Khandani, A.H. and Orlowski, R.Z. (2006) Bronchiolitis Obliterans with Organizing Pneumonia after Rituximab Therapy for Non-Hodgkin's Lymphoma. Hematological Oncology, 24, 234-237. https://doi.org/10.1002/hon.799

[6] Dhooria, S., Sehgal, I.S., Aggarwal, A.N., Behera, D. and Agarwal, R. (2016) Diagnostic Yield and Safety of TPC Probe Transbronchial Lung Biopsy in Diffuse Parenchymal Lung Diseases: Systematic Review and Meta-Analysis. Respiratory Care, 
61, 700-712. https://doi.org/10.4187/respcare.04488

[7] Wagner, S.A., Mehta, A.C. and Laber, D.A. (2007) Rituximab-Induced Interstitial Lung Disease. American Journal of Hematology, 82, 916-919.

https://doi.org/10.1002/ajh.20910

[8] Hiraga, J., Kondoh, Y., Taniguchi, H., Kinoshita, T. and Naoe, T. (2005) A Case of Interstitial Pneumonia Induced by Rituximab Therapy. International Journal of Hematology, 81, 169-170. https://doi.org/10.1532/IJH97.04163

[9] Roy, C., Felix, Z., Heidi, G. and Erhan, D.H. (2019) Transbronchial Lung Cryobiopsy in the Diagnosis of Interstitial Lung Disease: A Retrospective Single-Center Experience. Journal of Bronchology \& Interventional Pulmonology, 26, 15-21. https://doi.org/10.1097/LBR.0000000000000514

[10] American Thoracic Society and European Respiratory Society (2002) American Thoracic Society/European Respiratory Society International Multidisciplinary Consensus Classification of the Idiopathic Interstitial Pneumonias. American Journal of Respiratory and Critical Care Medicine, 165, 277-304.

https://doi.org/10.1164/ajrccm.165.2.ats01 


\section{Abbreviations}

RTX: Rituximab

P-ANCA: Perinuclear Anti-Neutrophilic Cytoplasmic Autoantibody

R-ILD: Rituximab Induced Interstitial Lung Disease

NSIP: Nonspecific Interstitial Pneumonia

GPA: Glomerulonephritis Granulomatosis with Polyangiitis

FVC: Forced Vital Capacity

TLC: Total Lung Capacity

DLCO: Diffusing Capacity

HSV: Herpes Simplex Virus

HZV: Herpes Zoster Virus

$\mathrm{H} \& \mathrm{E}=$ Hematoxylin and Eosin

Trichrome $=$ Masson's Trichrome Stain 